\title{
Quantitative Analysis of Ketoprofen Enantiomers by Liquid Chromatography/Electrospray Ionization-Mass Spectrometry
}

\author{
Fen YANG \\ Key Laboratory of Natural Resources of Changbai \\ Mountain and Functional Molecular (Department of \\ Chemistry, Yanbian University), Ministry of Education, \\ Yanji, China, \\ e-mail: 1255913130@qq.com
}

\author{
Dongri JIN* \\ Key Laboratory of Natural Resources of Changbai \\ Mountain and Functional Molecular (Department of \\ Chemistry, Yanbian University), Ministry of Education, \\ Yanji, China, \\ e-mail: drjin@ybu.edu.cn
}

\begin{abstract}
A enantioselective and sensitive liquid chromatography/ electrospray ionization-mass spectrometry (LC/ESI-MS) method is developed for the quantitative analysis of ketoprofen (KET) enantiomers based on a straightforward one-pot two-step stable isotope-coded derivatization of KET with a chiral derivatization reagent, (S)-1-methyl-4-(5-(3-aminopyrrolidin-1-yl)-2,4-dinitrophenyl)piperazine, and methyl iodide, respectively. The processes introduce not only a chiral tagging to the analytes but also a quaternary amine to the analytes, making the analytes permanently charged. The OPTSICD enabled the highly sensitive detection and complete separation of the KET enantiomers. By using second derivatization step with methyl iodide- $d_{3}\left(\mathrm{CD}_{3} \mathrm{I}\right)$, a complete set of deuterated standards was utilized and used as internal standards. The use of deuterated KET-derivatives significantly improved the assay precision and accuracy. The developed method was successfully applied to the quantitative analysis of KET in human saliva.
\end{abstract}

Keywords-ketoprofen; stable isotope-coded derivatization; LC/ESI-MS; carboxylic acid enantiomers; saliva

\section{INTRODUCTION}

Ketoprofen (KET) is a chiral non-steroidal antiinflammatory drug (NSAID) with analgesic and antipyretic actions. It inhibits cyclooxygenase (COX 1 and COX 2) activity with a reduction in tissue production of prostaglandins such as PGF2 $\alpha$ and $\mathrm{PGE}_{2}$. Nevertheless its therapeutic activity resides exclusively in the (S)enantiomer, it is still marked as racemic mixture [1]. Although NSAIDs are commonly used and believed to be safe, their acute overdose or chronic abuse sometimes causes serious side effects, such as gastrointestinal bleeding, intestinal ulceration, renal failure, hepatic failure, and aplastic anemia [2, 3]. Carefully monitoring the action of NSAIDs is therefore crucial with a view to improving the toxicological management of logn-term NSAIDs therapies. A number of High performance liquid chromatography (HPLC) [4-6] and capillary electrophoresis [7, 8] methods for the determination of KET enantiomers in biological fluids[9].

LC/ESI-MS (liquid chromatography/electrospray ionization-mass spectrometry) has emerged as a powerful method to detect carboxylic acid including NSAIDs. Generally, the carboxylic acid analytes were analyzed with LC/ESI-MS under negative ionization modes directly.
However, the best chromatographic resolution using a reversed-phase column is achieved at an acidic $\mathrm{pH}$, in which the deprotonation of the carboxyl group during the ionization process resulted in lower ionization efficiency and sensitivity [10]. To improve the MS response, carboxylic acids are frequently derivatized prior to analysis. Various derivatization reagents including chiral derivatization reagents for the MS response have been steadily developed for the labeling of carboxyl group [1114].

ESI (Electrospray ionization) is considered to be efficient for the detection of ionic molecules, but the ESI technique is prone to interference ion suppression from the matrix materials and/or co-eluting compounds during the LC/ESI-MS run. The ionization efficiency is also sometimes different between two single runs even for the same analyte. To overcome the matrix effects, the use of an isotope internal standard for the analyte often provides a high accuracy for quantitative determination. However, the stable isotope-labeled analytes were very expensive and its synthesis is not always easy. The stable isotope-coded derivatization (ICD) is an alternative way to introduce a stable isotope-coded moiety to the analyte, and the resulting derivative can be used as a substitute for a stable isotopelabeled internal standard (IS). Several ICD reagents [15, 16] have been developed for the quantitative analysis of carboxylic acids using LC/ESI-MS. However, the number of chiral ICD reagents for the LC/ESI-MS analysis is very limited. Higashi et al. introduced stable isotope-coded tags (DAPAP/d-DAPAP) into ibuprofen. The use of the isotopecoded reagent, $d$-DAPAP, significantly reduced the run-torun ionization differences, leading to a good assay precision and accuracy [17]. Although the method is excellent means for the quantification analysis, the disadvantages such as a pair of ICD reagents (light and heavy reagents labeled with $\mathrm{H} / \mathrm{D}$ ) is required and the isotope effects of H/D is inevitable.

We previously synthesized a novel chiral reagent, APyPPZ [18], which has two reaction groups (one is amine group; another is $\mathrm{N}$-methylpiperazine group). Here, we designed novel strategy that is to introduce a straightforward one-pot two-step stable isotope-coded derivatization (OPTSICD) where both samples (one is a biological sample containing KET, another is KET standard) were first derivatized with APy-PPZ to produce corresponding 
derivatives (KET-APy-PPZ), respectively, followed the produced derivatives are further separately converted to the precharged quaternary amine derivatives, KET-APy-MPPZ and $d$-KET-APy-MPPZ (as internal standard, IS), with commercial available $\mathrm{CH}_{3} \mathrm{I}$ and $\mathrm{CD}_{3} \mathrm{I}$, respectively. Both samples labeled with light and heavy are then mixed and subjected to the LC-MS analysis. The use of the isotopecoded derivative $d$-KET-APy-MPPZ (IS) significantly reduced the run-to-run ionization differences, leading to a good assay precision and accuracy.

\section{EXPERIMENTAL}

\section{A. Chemicals and Reagents}

Racemic KET and (S)-KET were purchased from Sigma-Aldrich (St. Louis, MO) and Dalian Meilun Biotech Co., Ltd. (China), respectively. 4-(4, 6-Dimethoxy-1, 3, 5triazin-2-yl)-4-methylmorpholinium chloride (DMT-MM) was from Tokyo Chemical Industry (Tokyo). Methyl iodide and methyl iodide- $d_{3}\left(\mathrm{CD}_{3} \mathrm{I}\right)$ were obtained from Aladdin (China). (S)-1-Methyl-4-(5-(3-aminopyrrolidin-1-yl)-2, 4dinitrophenyl)piperazine (APy-PPZ) was synthesized in our laboratories as previously reported [18].

\section{B. LC/ESI-MS}

The LC-ESI-MS systems consisted of an Agilent 1200 Series Rapid Resolution LC system and an Agilent 6410B Triple Quadrupole LC/MS system equipped with an electrospray source. Agilent MassHunter Workstation software version B.01.03 was used for data acquisition and qualitative analysis. Chromatographic separation was carried out at $313.15 \mathrm{~K}$ on a Mightysil RP-18 (GP) column $\left(3 \times 10^{-6} \mathrm{~m}, 150 \mathrm{~mm} \times 4.6 \mathrm{~mm}\right.$, i.d.). The mobile phase (gradient elution) consisted of acetonitrile (A)-water (B) containing $0.1 \% \mathrm{FA}$ aqueous solution at a flow rate of 0.3 $\mathrm{ml} / \mathrm{min}$. A gradient elution program was used with the ratio of A: B varied as follows: 0 s, 65:35; $900 \mathrm{~s}, 35: 65 ; 1020 \mathrm{~s}$, $5: 95$. The injection volume was $2 \mu \mathrm{L}$. The mass spectrometer was operated in positive mode. The optimized conditions consisted of a nebulizer pressure of $15 \mathrm{psi}$, a dry gas flow rate of $6 \mathrm{~L} / \mathrm{min}$ at $573.15 \mathrm{~K}$, an $\mathrm{HV}$ (high voltage) capillary voltage of $4000 \mathrm{~V}$, and a split voltage of $135 \mathrm{~V}$.

\section{Derivatization}

To a KET in methanol $(50 \mu \mathrm{L})$, solution of DMT-MM (1 $\mathrm{mM})$ in methanol $(50 \mu \mathrm{L})$ and APy-PPZ $(1 \mathrm{mM})$ in methanol $(50 \mu \mathrm{L})$ were successively added, then the mixture were reacted at $303.15 \mathrm{~K}$ on a heating block for $10 \mathrm{~min}$, followed by drying under nitrogen. To the dried residue was added $50 \mu \mathrm{L}$ of $\mathrm{CH}_{3} \mathrm{I}$ (or $\mathrm{CD}_{3} \mathrm{I}$ ), and the mixture was incubated at $333.15 \mathrm{~K}$ on a heating block for $1800 \mathrm{~s}$, then dried under nitrogen. To the residue was added $100 \mu \mathrm{L}$ of methanol prior to LC/ESI-MS analysis.

\section{RESULTS AND DISCUSSION}

\section{A. One-pot Two-step Stable Isotope-coded Derivatization (OPTSICD) of KET}

Fig. 1 shows the one-pot two-step stable isotope-coded derivatization reaction of KET with APy-PPZ and methyl iodide, respectively. KET enantiomers were first reacted with APy-PPZ at $313.15 \mathrm{~K}$ in DMT-MM as activation reagent for $600 \mathrm{~s}$, followed by drying under nitrogen to give the derivatezed KET (KET-APy-PPZ). The amounts of KET-APy-PPZ derivatives and KET that remained underivatized were monitored by HPLC-UV (detection at $254 \mathrm{~nm}$ ). The yields of the KET-APy-PPZ derivatives reached a plateau for $600 \mathrm{~s}$ and the underivatized KET was not detected. Thus, the first step derivatization reaction rapidly (600 s) proceeded under mild conditions (313.15 K). The KET-APy-PPZ derivatives in methnol were further converted to the perchaged quaternary amine derivatives (KET-APy-MPPZ or $d$-KET-APy-MPPZ) by reacting with methyl iodide $\left(\mathrm{CH}_{3} \mathrm{I}\right.$ or $\left.\mathrm{CD}_{3} \mathrm{I}\right)$ at $333.15 \mathrm{~K}$ for $1800 \mathrm{~s}$. This derivatization method is fast and quantitative, without a complicated handling procedure. The derivatization rate of pmol amounts of KET-APy-PPZ derivatives with $\mathrm{CH}_{3} \mathrm{I}$ was almost quantitative, because when KET-APy-PPZ (500 fmol) was derivatized, the KET-APy-PPZ was not detected by LC/ESI-MS (the minimum detectable amount of KETAPy-PPZ was $10 \mathrm{fmol} ; 2.0 \%$ of the initial amount).

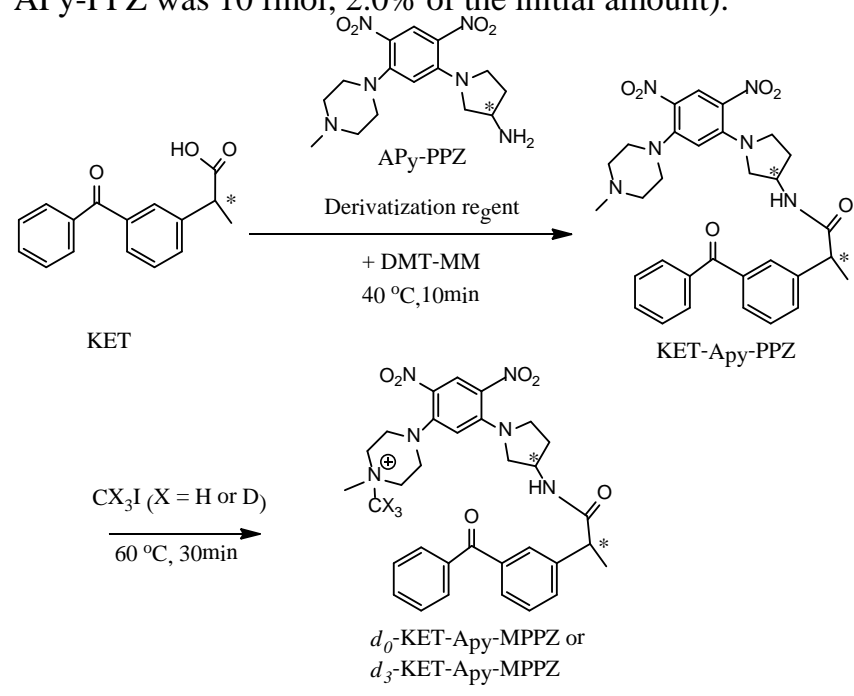

Figure 1. Derivatization reactions of KET with Apy-PPZ and methyl iodide, respectively. * indicates an asymmetric carbon.

\section{B. LC/ESI-MS Behavior of KET-APy-MPPZ and d-KET- APy-MPPZ Diastereomers}

The separation of the KET enantiomers after labeling with OPTSICD method was carried out by an ODS column packed with small particle silica-gel $\left(3 \times 10^{-6} \mathrm{~m}\right)$ using wateracetonitrile mixture containing $0.1 \% \mathrm{FA}$ as mobile phase. The detection of the separated diastereomers was performed by ESI-MS positive mode. The isocratic and gradient elution were examined to obtain the higher chromatographic resolution and higher ESI response of the diastereomers. Although the complete separation of (S)- and (R)-KET-APyMPPZ [retention times $\left(t_{R} \mathrm{~s}\right)$ of 670.2 and $711.6 \mathrm{~s}$, respectively, and resolution (Rs) of 4.3] was achieved by use of isocratic elution [water-acetonitrile containing $0.1 \%$ FA $(57: 43, v / v)]$, the gradient elution gave not only a better result for their separation [retention times $\left(t_{R} \mathrm{~s}\right)$ of 588 and $646.2 \mathrm{~s}$, respectively, and resolution (Rs) of 4.8] but also 
enhancement of ESI response. Fig. 2 shows typical SIM mass chromatograms of KET-APy-MPPZ/d-KET-APyMPPZ derivatives. As shown in Fig. 2, the KET-APyMPPZ/d-KET-APy-MPPZ derivatives are perfectly coeluted, which indicated no isotope effect associated with $d$-KET-APy-MPPZ derivatives. Although the correct reason of no isotope effect is not obvious, it seems to be due to prechaged quaternary amine moiety (hydrophilic group) in KET-APy-MPPZ which would avoid isotope effect.
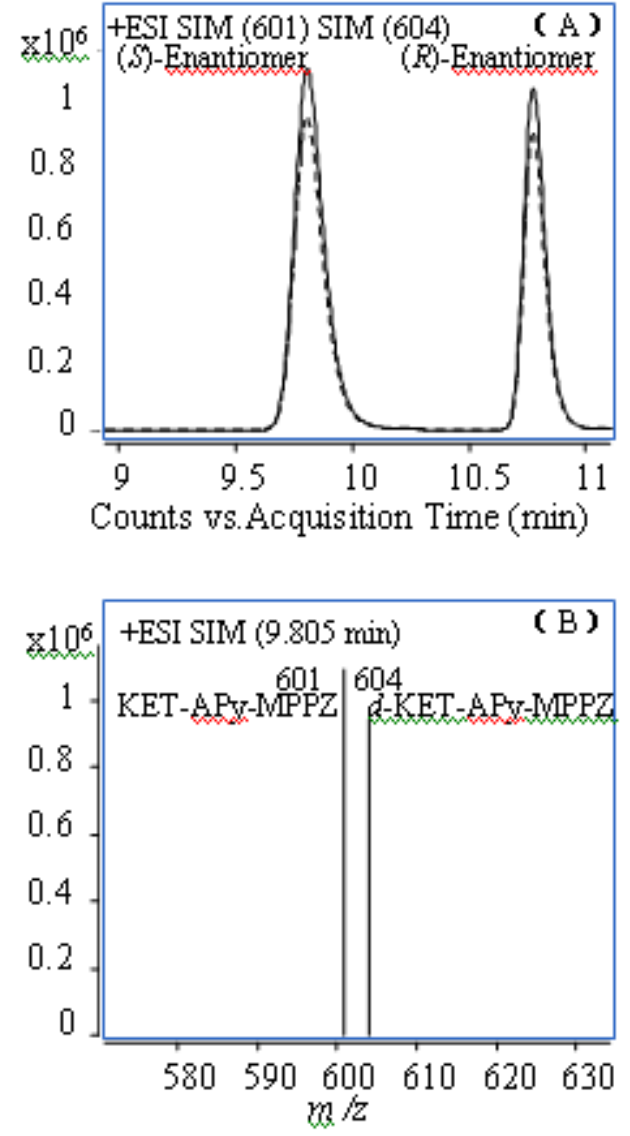

Figure 2. The SIM chromatogram monitored at $\mathrm{m} / \mathrm{z} 601$ (full line) and 604 (dotted line) (A) and ESI-MS spectrum (B).

The mass spectrometric features of KET-APy-MPPZ diastereomers were also investigated using the LC/ESI-MS (/MS). For the LC/ESI-MS operation in the positive ion mode, KET-APy-MPPZ diastereomers gave only their molecular cations, $\left[\mathrm{M}^{+}\right]$, without any fragment ions. The SIM mode of the monitoring $\left[\mathrm{M}^{+}\right]$of the KET-APy-MPPZ was used for the evaluation of the effect of the OPTSICD method.

\section{Method Validation}

The calibration curves for both $(S)$-KET, and $(R)$-KET were linear over the concentration range of 1.0-100 $\mathrm{nmol} / \mathrm{L}$ with determination coefficients $\left(r^{2}\right)$ of greater than 0.999 for both enantiomers. Linear regression equation were

$$
Y=0.2999 X+2.0537
$$

Equation (1) is for (S)-KET,

$$
Y=0.24508 X+2.34549
$$

Equation (2) is for (R)-KET;

Where $Y$ represents the ratio of sample area and internal standard area, $X$ represents concentration. The detection limits were $0.1 \mathrm{nmol} / \mathrm{L}$ for $(S)$-KET and $(R)$-KET, respectively, with $\mathrm{S} / \mathrm{N}$ ratios $=3$.

Method validation regarding intra-day precision assays was achieved by replicate injection of saliva sample of the analytes at three different concentration levels (1.0, 10.0, $50.0 \mathrm{nmol} / \mathrm{L}$ ) within the calibration curve range. The relative standard deviation (RSD) $(n=5)$ was calculated for the ratio of sample area and internal standard area and found to be $<5.3 \%$ in all cases.

The assay accuracy was evaluated as the analytical recovery. As shown in Table $\mathrm{I}$, satisfactory recovery rates ranging from 105.2 to $108.2 \%$ were obtained. These data indicated that the present method is highly accurate.

\begin{tabular}{|c|c|c|c|c|}
\hline & \multirow{2}{*}{$\begin{array}{c}\text { (S)-KET } \\
\text { Measured }^{\mathrm{a}} \\
(\mathrm{ng} / \mathrm{mL})\end{array}$} & \multicolumn{3}{|c|}{$(R)-\mathrm{KET}$} \\
\hline & & $\begin{array}{c}\text { Accuracy }^{\mathrm{b}} \\
(\%)\end{array}$ & $\begin{array}{l}\text { Measured }^{\mathrm{a}} \\
(\mathrm{ng} / \mathrm{mL})\end{array}$ & $\begin{array}{c}\text { Accuracy } \\
(\%)\end{array}$ \\
\hline $\begin{array}{l}\text { Saliva }^{\mathrm{c}} \\
\text { (intact) }\end{array}$ & 1.23 & - & 1.07 & - \\
\hline $\begin{array}{l}\text { Spiked } \\
\text { sample } \\
(+1.20 \\
\mathrm{ng} / \mathrm{mL})\end{array}$ & 2.63 & 108.2 & 2.40 & 107.4 \\
\hline $\begin{array}{c}\text { Spiked } \\
\text { sample } \\
(+2.50 \\
\text { ng/mL })\end{array}$ & 3.93 & 105.2 & 3.79 & 106.2 \\
\hline \multicolumn{5}{|c|}{${ }^{a}$ Mean ( $\mathrm{n}=5$ for intact sample and $\mathrm{n}=3$ for spiked sample) } \\
\hline
\end{tabular}

TABLE I. ASSAY ACCURACY OF $(S) /(R)$-KET FOUND IN HUMAN SALIVA

\section{CONCLUSION}

We have demonstrated a one-pot two-step isotope-coded derivatization method for the enantioselective determination of KET in saliva. This method employed derivatization with the chiral reagent, APy-PPZ which is able to be quarternarized, not only to increase the detectability of KET in the positive ESI-MS, but also to introduce an isotope moiety to the derivatives. The use of the isotope-coded derivatives, $d_{3}$-KET-APy-MPPZ, significantly reduced the run-to-run ionization differences, leading to a good assay precision and accuracy. To the best of our knowledge, this is the first LC/MS assay that enables the enantioselective quantification of salivary KET. The developed method will be useful in clinical and basic research studies not only for 
KET, but also for other carboxylic acid containing chiral drugs, whose isotope-labeled ISs and its isotope-labeled derivatization reagents are difficult to prepare.

\section{ACKNOWLEDGMENT}

The present research was supported in part by the National Natural Science Foundation of China (21365022, 21565027).

\section{REFERENCES}

[1] A. Boobis, M. Rawlins, S. Thomas, M. Wilkins, in: C. Dollery (Ed.), The Drugs, Churchill Livingstone, Edinburgh, 1999, p. 18.

[2] María Victoria Toledo a , Carla José a , Sebastián E. Collins b , María Luján Ferreira c ,Laura E. Briand, "Towards a green enantiomeric esterification of R/S-ketoprofen: A theoretical and experimental investigation”, Journal of Molecular Catalysis B: Enzymatic .118 ,2015, pp.52-61.

[3] P. Magill, P.F. Ridgway, K.C. Conlon, P. Neary, "A caseof probable ibuprofeninduced acute pancreatitis”, J. Pancreas 7 ,2006,pp. 311314.

[4] F.J. López-Muñoz, N.V. Gama, O. Soria-Arteche, M.H. Peña, A.M. Domínguez-Ramírez, J.R.M. López, "HPLC method with solid-phase extraction for determination of (R)-and (S)-ketoprofen in plasma without caffeine interference: Application to pharmacokinetic studies in rats”, J. Chromatogr. Sci. 52, 2014, pp. 1204-1210.

[5] Y. X. Jin, Y.H. Tang, S. Zeng, "Analysis of flurbiprofen, ketoprofen and etodolac enantiomers by pre-column derivatization RP-HPLC and application of drug-protein binding in human plasma”, J. Pharm. Biomed. Anal. 46, 2008, pp. 953-958.

[6] Juan M. Padró, Jaiver Osorio-Grisales, Juan A. Arancibia, Alejandro C. Olivieri, Cecilia B. Castells. "Scope of partial least-squares regression applied to the enantiomeric composition determination of ketoprofen from strongly overlapped chromatographic profiles", Journal of separation science.38, 2015, pp. 2423-2430.

[7] F.K. Glówka, "Determination of ketoprofen enantiomers in human serum by capillary zone electrophoresis: man pharmacokinetic studies after administration of rac-kettoprofen tablets”, J. Pharm. Biomed. Anal. 30, 2002, pp. 1035-1045.

[8] F.K. Glówka, M. Karaźniewicz, "High performance capillary electrophoreis for determination of the enantiomers of 2-arylpropionic acid derivatives in human serum pharmacokinetic studies of ketoprofen enantiomers following administration of standard and sustained release tablets”, J. Pharm. Biomed. Anal. 35, 2004, pp. 807816.
[9] Sangyoung Yoon, Seungbum Kim, Songyi Park, Eunsoo Hong, Jinyeong Kim, et al. "Improving the enantioselectivity of an esterase toward (S)-ketoprofen ethyl ester through protein engineering", Journal of Molecular Catalysis B: Enzymatic. 100, 2014, pp. 25-31.

[10] M. Jemal, O. Zheng, D. Teiz, "High performance liquid chromatography mobile phase composition optimization for the quantitative determination of a carboxylic acid compound in human plasma by negative ion electrospray high performance liquid chromatography tandem mass spectrometry”, Rapid Commun. Mass Spectrom. 12, 1998, pp. 429-434.

[11] T. Higashi, T. Ichikawa, S. Inagaki, J.Z. Min, T. Fukushima, T. Toyo'oka, "Simple and practical derivatization procedure for enhanced detection of carboxylic acids in liquid chromatographyelectrospray ionization-tandem mass spectrometry", J. Pharm. Biomed. Anal. 52, 2010, pp. 809-818.

[12] H. Tsutsui, S. Fujii, T. Sakamoto, J.Z. Min, K. Todoroki, T. Toyo'oka, "Chiral amines as reagents for HPLC-MS enantioseparation of chiral carboxylic acids”, J. Sep. Sci. 35, 2012, pp. 1551-1559.

[13] T. Higashi, M. Kawasaki, H. Tadokoro, S. Ogawa, H. Tsutsui, T. Fukushima, T. Toyo'oka, "Derivatization of chiral carboxylic acids with (S)-anabasine for increasing detectability and enantiomeric separation in LC/ESI-MS/MS”, J. Sep. Sci. 35, 2012, pp. 2840-2846.

[14] S. Ogawa, H. Tadokoro, M. Sato, T. Hanawa, T. Higashi, “(S)-1-(4Dimethylaminophenylcarbonyl)-3-aminopyrrolidine: A derivatization reagent for enantiomeric separation and sensitive detection of chiral carboxylic acids by LC/ESI-MS/MS”, J.Chromatogr. B, 940, 2013, pp. 7-14.

[15] T. Toyo'oka, "LC-MS determination of bioactive molecules based upon stable isotope-coded derivatization method", J. Pharm. Biomed. Anal. 69, 2012, pp. 174-184.

[16] P. Bruheim, H.F.N. Kvitvang, S.G. Villas-Boas, "Stable isotope coded derivatizing reagents as internal standards in metabolite profiling”, J. Chromatogr. A 1296, 2013, pp. 196-203.

[17] S. Ogawa, H. Tdokoro, M. Sato, T. Higashi, "Enantioselective determination of ibuprofen in saliva by liquid chromatography/tandem mass spectrometry with chiral electrospray ionization-enhancing and stable isotope-coded derivatization”, J. Pharm. Biomed. Anal. 98, 2014, pp. 387-392.

[18] D Jin, Y. Zhao, Z. Z. Min, Y.I. Lee, “(S)-1-methyl-4-(5-(3aminopyrrolidin-1-yl)-2, 4 dinitrophenyl) piperazine as a novel chiral derivatization reagent for high-performance liquid chromatographic analysis of carboxylic acid enantiomers”, Microchem.J. 118, 2015, pp. 176-182. 